\title{
CONTAMINACIÓN ORGÁNICA DEL RÍO CHAMBO EN EL ÁREA DE DESCARGA DE AGUA RESIDUAL DE LA CIUDAD DE RIOBAMBA
}

\section{Organic Contamination of The Chambo River in The Wastewater Discharge Area of The City of Riobamba}

\author{
${ }^{1}$ Jaime Béjar Suárez*, ${ }^{2}$ Benito Mendoza Trujillo* \\ ${ }^{1}$ Escuela Superior Politécnica de Chimborazo, Panamericana Sur km 1 1/2, \\ Riobamba-Ecuador. \\ ${ }^{2}$ Laboratorio de Servicios Ambientales, Universidad Nacional de Chimborazo, \\ Riobamba, Ecuador. \\ bejarjaime@gmail.com / benitomendoza@unach.edu.ec
}

\begin{abstract}
$\mathbf{R}$
El problema de la contaminación de las aguas es uno de los grandes desafíos para la humanidad. Por lo tanto, su estudio y conocimiento es una necesidad, mas aún en lugares con poca o ninguna infraestructura que permita el tratamiento de aguas residuales, tal como es el caso del vertimiento directo de aguas servidas en la mayoría de los ríos del Ecuador. Esta investigación busca determinar la contaminación orgánica en un tramo del río Chambo, localizado en la provincia de Chimborazo, Ecuador, en el que se vierten, sin tratamiento previo las aguas residuales de la ciudad de Riobamba, así como las aguas contaminadas del río Chibunga. Para este estudio se aplica el índice Icomopara la valoración de la contaminación orgánica en ríos, basado en la determinación experimental del oxígeno disuelto, $\mathrm{DBO}_{5}$ y coliformes totales. Las mediciones realizadas de estos parámetros fueron realizadas en un espacio temporal de 7 días en 7 puntos de muestreo. El estudio verificó que el río mantiene un nivel de contaminación orgánica bajo antes de la descarga de agua residual y que además después de la misma logra auto-depurarse aguas abajo por efecto de la geomorfología y el caudal que este posee.
\end{abstract}

Palabras claves: río Chambo, río Chibunga, Icomo, contaminación orgánica.

\section{A bstract}

The problem of water pollution is one of the great challenges for humanity. Therefore, its study and knowledge are needed, especially in places with little infrastructure that allows the treatment of wastewater, such as the case of direct dumping of wastewater in most of the Ecuadorean rivers. The purpose of this research is to determine organic contamination in a section of the Chambo River, located in the province of Chimborazo-Ecuador, where it is discharged, without considering the wastewater from the city of Riobamba, as well as the contaminated waters from the Chibunga River. For this study, the Icomo index is applied for the assessment of organic pollution in rivers, based on the experimental determination of dissolved oxygen, $\mathrm{BOD}_{5}$ and total coliforms. The measurements took place in a temporary space of 7 days in 7 sampling points. The study made it possible to verify that the river remains at a low level of organic contamination before the discharge of wastewater and that afterwards it was able to automatically purify the downstream waters due to the effect of the geomorphology and its flow

Keywords: Chambo river, Chibunga river, Icomo, organic contamination. 


\section{INTRODUCCIÓN}

La contaminación del agua es un problema relacionado con el crecimiento de la población y diversas actividades del hombre, junto a procesos naturales. Pero las principales causas de la contaminación del agua son las actividades antrópicas de grandes asentamientos humanos (1). En Latinoamérica, las descargas de aguas residuales urbanas están aumentando debido a: 1) el crecimiento de la población, pues la población urbana ha aumentado de 314 millones en 1990 a casi 496 millones en la actualidad, y se prevé que llegará a 674 millones para el año 2050 (2); y 2) expansión del agua de suministro y saneamiento. En la mayor parte de la región, las aguas residuales urbanas son una preocupación (3).

Los efectos de la contaminación de los ríos crearon la necesidad de desarrollar métodos que permiten medir el nivel de contaminación de estos, basados en la medición experimental de un conjunto de parámetros y operaciones matemáticas que van desde operaciones aritméticas básicas, hasta complicados modelos matemáticos. De ahí que el monitoreo y el reporte del grado de contaminación del agua, en particular de los ríos, sea de gran importancia para lograr la elaboración de estrategias y políticas ambientales. En otras palabras, si no se logra medir el grado de contaminación, no se puede definir el problema de la contaminación y consecuentemente la eficacia de políticas ambientales no pueden ser implementadas (4).

Es así que se crearon los Indices de Calidad de Agua (ICA) (5) los mismos que fueron una adaptación del Water Quality Index (WQI), este índice utiliza una valoración de 0 a 100 para calificar la calidad del agua utilizando 10 parámetros como oxígeno disuelto (OD), coliforme fecales, potencial hidrógeno $(\mathrm{pH})$, demanda biológica de oxígeno $\left(\mathrm{DBO}_{5}\right)$, temperatura, fosfatos, nitritos, turbidez y sólidos totales (6); la aplicación del
ICA es muy bueno cuando se cuenta con recursos económicos, ya que realizar el análisis de todos los parámetros en muchos puntos de monitoreo resulta costoso, además de que se ha demostrado que no tiene mucha coherencia con los resultados de índices biológicos (7). De aquí resulta que el índice utilizado en este trabajo para medir la contaminación orgánica y denominado Icomo es conveniente al depender únicamente de tres parámetros (7), además que se ha demostrado que sus valores tienen similitud con otros indicadores como el ICA-NSF propuesto por la Fundación Nacional de Saneamiento de los Estados Unidos (8).

El río Chambo es el río principal de la subcuenca del mismo nombre ubicado en la zona central del Ecuador. De acuerdo con lo expuesto por la Central Ecuatoriana de Servicios Agrícolas (2015) el promedio anual del caudal del río Chambo es de $62 \mathrm{~m}^{3} / \mathrm{s}$ de los cuales por el tipo de consumo sus porcentajes de consumo son: $71,91 \%$ riego y abrevadero, 9,56\% consumo doméstico, $0,28 \%$ Piscicultura, 9,09\% Hidroelectricidad, 5,14\% Industrial y $4,02 \%$ recreativo.

En este contexto, el área de estudio comprende un tramo de cerca de $1 \mathrm{~km}$ del río Chambo, en el que se realiza la valoración de la contaminación orgánica en el mencionado tramo en un espacio temporal de 7 días, de tal manera que permita valorar la utilización del índice ICOMO para determinar el nivel de contaminación orgánica con respecto al tiempo.

\section{MATERIAL Y MÉTODOS}

\section{Descripción del área de estudio}

El área de estudio escogida dentro de la subcuenca del río Chambo es $10 \mathrm{~m}$ antes de la desembocadura del río Chibunga en el río Chambo hasta $977 \mathrm{~m}$ aguas abajo del mismo. Este tramo de estudio se escogió porque cuenta con las siguientes características: aguas arriba de la desembocadura no existe mayor afección antrópica, después se tiene la desembocadura del río Chibunga el mismo que presenta afección antrópica ya que pasa por un lado de la ciudad y tiene varias descargas de agua residual a lo largo del mismo, por lo que puede presentar características de un río polucionado (9); algunos metros más abajo se encuentra la descarga de agua residual de la ciudad de Riobamba, la misma que no presenta tratamiento previo, lo que cambia las condiciones del río; y se cierra el tramo en el puente de acceso a Chambo para verificar cómo cambia la concentración de los contaminantes en el río. 


\section{El índice de contaminación por materia orgánica Icomo}

El índice de contaminación por materia orgánica (Icomo) se expresa mediante el promedio de otros tres índices que dependen respectivamente de: la demanda bioquímica de oxígeno $\left(\mathrm{DB}_{5}\right)$, coliformes totales y porcentaje de saturación del oxígeno (7):

La manera cómo se determina este índice es mediante

$$
\mathrm{ICOMO}=\frac{1}{3}\left(\mathrm{I}_{\mathrm{DBO}}+\mathrm{I}_{\text {Coliformes totales }}+\mathrm{I}_{\% \text { Oxígeno }}\right)
$$

el cálculo independiente de los índices componentes del Icomo de acuerdo con las siguientes expresiones (7):

$I_{\text {DвО }}$ se obtiene de la siguiente expresión:

$$
\mathrm{I}_{\mathrm{DBO}}=-0,05+0,70 \log _{10} \quad \mathrm{DBO}(\mathrm{mg} / \mathrm{L})
$$

$\mathrm{I}_{\text {Coliformes totales }}$ se obtiene de la siguiente expresión:

$$
\text { Si } D B O>30 \frac{\mathrm{mg}}{\mathrm{L}} \Rightarrow \mathrm{I}_{\mathrm{DBO}}=1 \text { ysi } D B O<2 \frac{\mathrm{mg}}{\mathrm{L}} \Rightarrow \mathrm{I}_{\mathrm{DBO}}=0
$$

$I_{\% \text { Oxígeno }}$ se obtiene de la siguiente expresión:

$$
\mathrm{I}_{\text {Coliformes }}=-1,44+0,56 \log _{10} \text { Col.Tot. }\left(\frac{\mathrm{NMP}}{100 \mathrm{~mL}}\right)
$$

$$
\begin{aligned}
& \text { Si Col. Tot. }>2000 \frac{\mathrm{NMP}}{100 \mathrm{~mL}} \Rightarrow \mathrm{I}_{\text {Col. Tot. }}=1 \\
& y \text { si Col. Tot }<500 \frac{\mathrm{NMP}}{100 \mathrm{~mL}}=>\mathrm{I}_{\text {Coliformes }}=0 \\
& \mathrm{I}_{\% \text { Oxígeno }}=1-0,01 \% \mathrm{OD} \\
& \text { Si } \% \text { OD }>100 \% \Rightarrow \mathrm{I}_{\% \text { Oxígeno }}=0
\end{aligned}
$$

\section{Criterios para la aplicación del índice Icomo}

Según el intervalo en que se encuentre un valor dado del índice Icomo se identifica el nivel de contaminación orgánica del agua (7), tal como muestra el Cuadro 1. Aquí se observa que la clasificación de la contaminación es: contaminación muy baja, contaminación baja, contaminación media, contaminación alta y contaminación muy alta. Para el estudio se toman muestras durante 7 días consecutivos en 7 puntos diferentes; estos puntos se encuentran separados entre el primero y el último una distancia de $977 \mathrm{~m}$. A fin de que la muestra sea representativa se toman diariamente 3 muestras (09:00, 13:00 y 16:00). De estos puntos se obtienen valores medios diarios de $\mathrm{DBO}_{5}$, coliformes totales, y concentración de oxígeno disuelto, además de caudales, profundidad, temperatura, presión atmosférica y velocidad de la corriente. Con estos datos se identifican los niveles de contaminación orgánica.

Para el análisis de laboratorio se realizó una muestra compuesta por día para el análisis de $\mathrm{DBO}_{5}$ y coliformes totales en el en el Laboratorio de Servicios Ambientales. Este laboratorio cuenta con la acreditación por el Sistema de Acreditación Ecuatoriano (SAE) y utiliza como técnicas de laboratorio las de los Métodos Estándar para Análisis de Agua y Agua residual, edición 21.

\section{RESULTADOS Y DISCUSIÓN}

Los 7 puntos de muestreo comprenden una longitud de $977 \mathrm{~m}$ del río Chambo según se describen en el cuadro 2 .

Los resultados del muestreo presentan valores medios de la medición in situ, en la Figura 1, se muestra como van variando los valores de $\% \mathrm{OD}, \mathrm{DBO}_{5}$ y coliformes totales. El comportamiento de estas variables en el tramo del río Chambo muestra que a pesar de que los valores de estos parámetros son diferentes en cada día de monitoreo, cumplen una tendencia por la presencia de la desembocadura del río Chibunga y la presencia de la descarga de agua residual de la ciudad de Riobamba. Obsérvese que los mínimos en la figura 1 a) corresponden a los puntos inmediatamente posteriores 


\begin{tabular}{|c|c|c|}
\hline Definición & Valor del ICOMO & Nivel de contaminación \\
\hline \multirow{5}{*}{$\mathrm{ICOMO}=\frac{1}{3}\left(\mathrm{I}_{\mathrm{DBO}}+\mathrm{I}_{\text {Coliformes totales }}+\mathrm{I}_{\% \text { Oxígeno }}\right)$} & $0 \leq \mathrm{ICOMO} \leq 0,2$ & Contaminación muy baja \\
\hline & $0,2 \leq \mathrm{ICOMO} \leq 0,4$ & Contaminación baja \\
\hline & $0,4 \leq \mathrm{ICOMO} \leq 0,6$ & Contaminación media \\
\hline & $0,6 \leq \mathrm{ICOMO} \leq 0,8$ & Contaminación alta \\
\hline & $0,8 \leq \mathrm{ICOMO} \leq 0,10$ & Contaminación muy alta \\
\hline
\end{tabular}

Cuadro 1. Criterios sobre el índice ICOMO, basados en (10)

a las confluencias de las descargas del río Chibunga y del vertimiento de las aguas servidas de la ciudad de Riobam$\mathrm{ba}$, mientras que en la figura $1 \mathrm{~b}$ ) y c) los puntos máximos corresponden, respectivamente con los puntos inmediatamente posteriores a dichas descargas.

En la figura 2 se muestra cómo varía el Icomo en cada día de monitoreo, de acuerdo al punto de muestreo descrito anteriormente.

\begin{tabular}{|c|c|c|c|}
\hline Código & X & Y & Altitud \\
\hline $\mathrm{CH} 1$ & 764963 & 9809892 & 2609 \\
\hline $\mathrm{CH} 2$ & 765102 & 9810088 & 2608 \\
\hline $\mathrm{CH} 3$ & 765379 & 9810242 & 2605 \\
\hline $\mathrm{CH} 4$ & 765560 & 9810351 & 2605 \\
\hline $\mathrm{CH} 5$ & 765567 & 9810369 & 2604 \\
\hline $\mathrm{CH} 6$ & 765625 & 9810450 & 2602 \\
\hline $\mathrm{CH} 7$ & 766054 & 9810581 & 2596 \\
\hline
\end{tabular}

Cuadro 2. Ubicación geográfica de los puntos de muestreo en el tramo del río Chambo

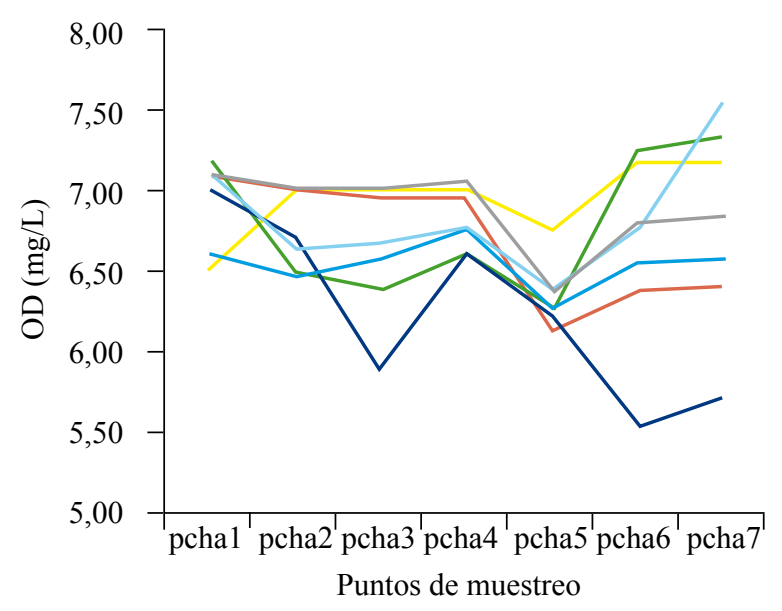

(a)

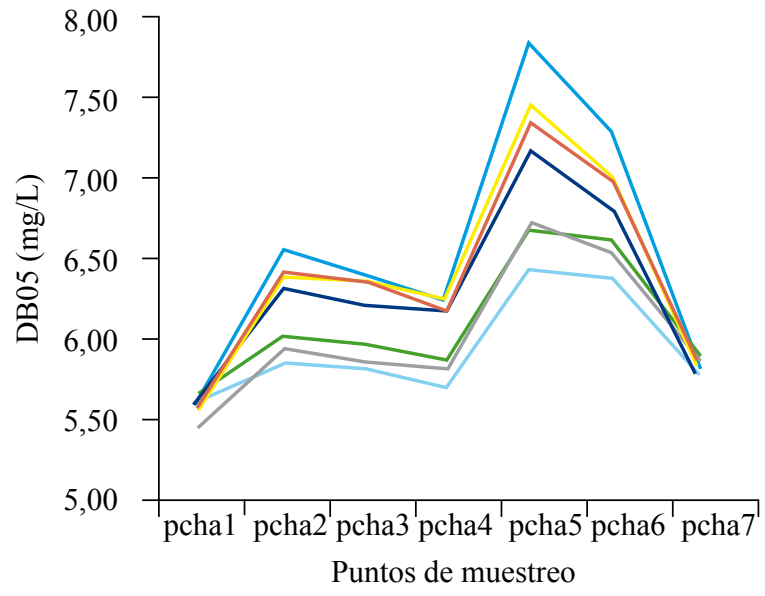

(b)

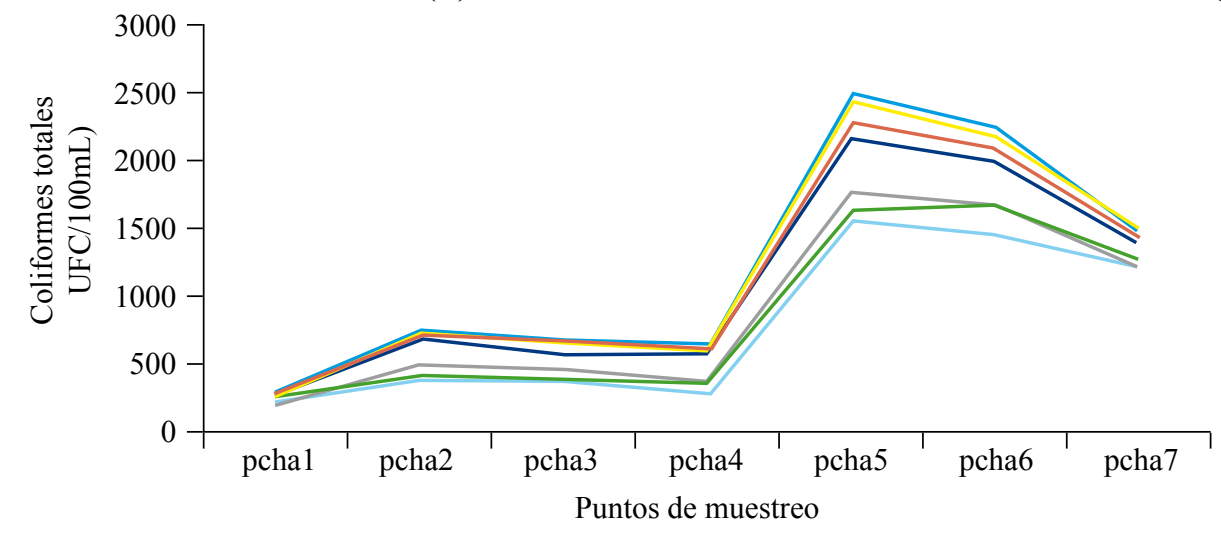

$10 / 10 / 2017$

$11 / 10 / 2017$

$12 / 10 / 2017$

13/10/2017

$14 / 10 / 2017$

$16 / 10 / 2017$

$17 / 10 / 2017$

(c)

Figura 1. Comportamiento del $\mathrm{OD}(\mathrm{a}), \mathrm{DBO}_{5}$ (b) y coliformes totales (c) en los puntos de muestreo en el tramo del río Chambo. 



Figura 2. Comportamiento del OD (a), DBO5 (b) y coliformes totales (c) en los puntos de muestreo en el tramo del río Chambo.

Realizando una valoración media del índice Icomo en los 7 días de muestreo (figura 3) se observa que: el punto $\mathrm{CH} 1$ (valor medio 0,24 ) muestra una contaminación baja, ya que no se tiene mayor influencia antrópica. El punto $\mathrm{CH} 2$ muestra también baja contaminación, es decir que el río Chibunga no aporta de gran manera contaminantes orgánicos al río Chambo, los puntos $\mathrm{CH} 3$ y $\mathrm{CH} 4$ que se encuentran antes de la descarga de agua residual presentan un valor medio de 0,32 , es decir contaminación orgánica baja por no existir influencia antrópica de ningún tipo en este tramo. En el punto $\mathrm{CH} 5$ se ve ya la influencia de la carga contaminante de la descarga, en este punto se observa un índice con valor medio de 0,58 que corresponde a contaminación orgánica media; en el punto $\mathrm{CH} 6$ aguas abajo se observa que se mantiene el índice (valor medio 0,57 ) de contaminación orgánica en medio, se evidencia que aquí aún existe influencia de la descarga en el río y este no logra auto-depurarse. En el punto $\mathrm{CH} 7$ el índice (valor medio 0,38) muestra un nivel de contaminación orgánica bajo, aquí el río por efectos de su caudal, que en el período de monitoreo fue de $24 \mathrm{~m}^{3} / \mathrm{s}$, y de la pendiente de $22 \%$ (11) logran de alguna manera auto-depurar el río.

\section{CONCLUSIONES}

Las concentraciones de $\mathrm{OD}, \mathrm{DBO}_{5}$ y coliformes totales en los puntos de muestreo muestran variaciones a lo largo de los puntos de muestreo, encontrándose que las concentraciones de $\mathrm{DBO}_{5}$ y co- 


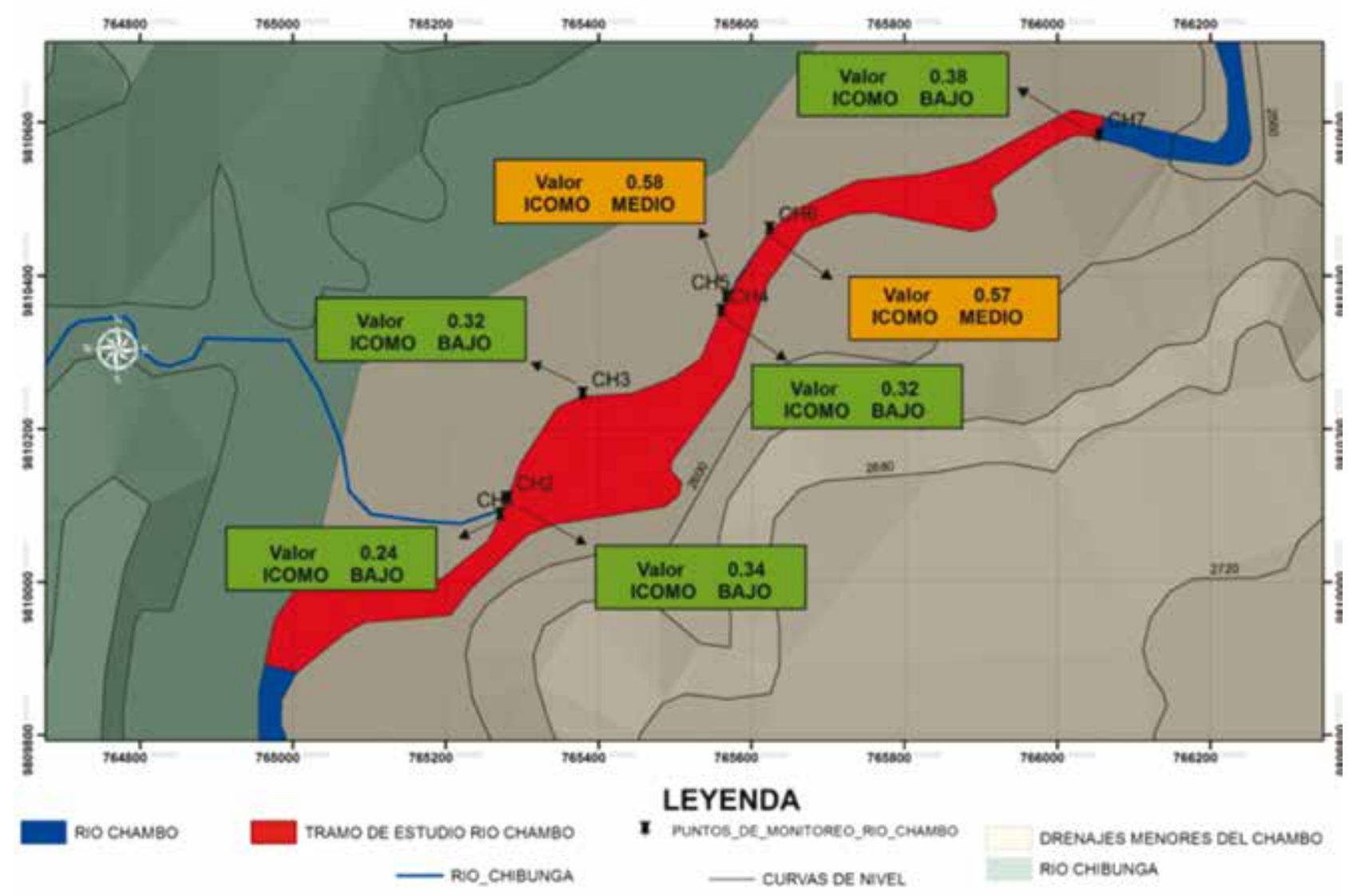

Figura 3. Valores de medios del ICOMO en los puntos de muestreo en el tramo del río Chambo.

liformes totales aumentan después del ingreso de cargas contaminantes al río mientras que con OD, ocurre lo contrario.

El índice Icomo permitió determinar la contaminación orgánica en el tramo del río Chambo, y la selección de este permitió determinar resultados de contaminación baja en los puntos donde no existe intervención antrópica y valores altos en puntos que tienen contaminación orgánica como la descarga de agua residual de la ciudad de Riobamba.

Se evidencia como el río Chambo por sus condiciones geomorfológicas y de caudal logra auto depurarse a medida que el agua residual de la ciudad de Riobamba se mezcla con el agua del río, y que por efecto de la geomorfología del río en este sector, es decir pendiente y configuración rocosa, esta recupera los valores de oxígeno disuelto, estas características permiten descomponer la materia orgánica a las bacterias. Aún así, exista esta autodepuración es necesario que las autoridades de la ciudad de Riobamba, realicen el tratamiento de las aguas residuales que desembocan tanto en el río Chibunga como en el río Chambo, para de esta manera cumplir con la Norma Ambiental vigente en el país, y brindar a la ciudadanía un ambiente sano. 
1. United Nations. Policy Brief on Water Quality. Ontario:; 2011.

2. United Nations. World urbanization prospects: The 2014 revision, Highlights New York: Department of Economic and Social Affairs; 2014.

3.Connor R, Renata A, Ortigara C, Koncagül E, Uhlenbrook S, Lamizana-Diallo BM, et al. The United Nations World Water Development Report 2017: Wastewater, The Untapped Resource. Paris:; 2017.

4. Lamizana-Diallo BL, Salinas A, Tonda E, Rabbiosi L, Milà i Canals L, Spencer D. Preventing and reducing wastewater generation and pollution loads at the source. In UNESCO. Wastewater: the untapped resource. Paris: United Nations Educational, Scientific and Cultural Organization; 2017. p. 198.

5. Martínez de Bascaran G. El índice de calidad del agua. Ingeniería Química. 1976;: p. 45-49.

6. Cude C. Oregon water quality index a tool for evaluating water quality management effectiveness. JAWRA Journal of the American Water Resources Association. 2001; 37(1): p. 125-137.

7. Ramírez A, Restrepo R, Viña G. Cuatro índices de contaminación para caracterización de aguas continentales. Formulaciones y aplicación. CT\&F-Ciencia, Tecnología y Futuro. 1997; 1(3): p. 135-153.

8. Valdes J, Samboni N, Carvajal Y. Desarrollo de un Indicador de calidad de agua usando estadística aplicada, caso de estudio: subcuenca Zanjón Oscuro. Tecno Lógicas. 2011;(26): p. 165-180.

9. Jaque Castellano E, Guerrero P, Lucia C. Evaluación del índice de calidad de agua (ica) de la microcuenca del río chibunga, en variaciones estacionales, provincia de chimborazo-ecuador, durante el periodo 2014. 2015..

10. Samboni NE, Reyes T. A, Carbajal E. Y. Aplicación de los indicadores de calidad y contaminación. Ingeniería y Competitividad. 2011; 13(2): p. 49-60.

11. Mendoza B. Characterization of real aquifers using hydrogeophysical measurements. An application to the Chambo aquifer (Ecuador). 2015 Marzo.. 